\title{
Medical Imaging for the Tracking of Micromotors
}

D. Vilela ${ }^{1,2}$, U. Cossio ${ }^{4}$, J. Parmar ${ }^{1,2}$, A.M. Martínez-Villacorta ${ }^{4}$, V. Gómez-Vallejo ${ }^{4}$, J. Llop ${ }^{4}$ and S. Sánchez ${ }^{1,2,3 *}$

${ }^{1}$ Institute for Bioengineering of Catalonia (IBEC), The Barcelona Institute of Science and Technology, Baldiri Reixac 10-12, 08028 Barcelona Spain

${ }^{2}$ Max Planck Institute for Intelligent Systems Institution, Heisenbergstraße 3, 70569 Stuttgart, Germany.

${ }^{3}$ Institució Catalana de Recerca i Estudis Avancats (ICREA), Pg. Lluís Companys 23, 08010, Barcelona, Spain

${ }^{4}$ Radiochemistry and Nuclear Imaging Group, CIC biomaGUNE, Paseo Miramón 182, 20014, San Sebastián, Spain

*Corresponding Authors: Prof. S. Sánchez (ssanchez@ibecbarcelona.eu)

\section{ABSTRACT}

Micro/nanomotors are useful tools for several biomedical applications, including targeted drug delivery and minimally invasive microsurgeries. However, major challenges such as in vivo imaging need to be addressed before they can be safely applied on a living body. Here, we show that positron emission tomography (PET), a molecular imaging technique widely used in medical imaging, can also be used to track a large population of tubular Au/PEDOT/Pt micromotors. Chemisorption of an Iodine isotope onto the micromotor's Au surface rendered them detectable by PET and we could track their movements in tubular phantom over time frames of up to 15 minutes. In a second set of experiments, micromotors and the bubbles released during selfpropulsion were optically tracked by video imaging and bright field microscopy. The results from direct optical tracking agreed with those from PET tracking, demonstrating that PET is a suitable technique for the imaging of large populations of active micromotors in opaque environments, thus opening opportunities for the use of this mature imaging technology for the in vivo localization of artificial swimmers. 
Keywords: micromotors, medical imaging, microswimmers, microjets, PET imaging, microbots.

Micro/nanomotors are micro/nanoscale devices which are capable of generating small scale autonomous motion in fluid environments ${ }^{1}$. Over the last decade, this field has attracted increasing scientific interest, and, as a consequence, impressive progress has been made in developing a variety of micro/nanoscale motors with different propulsion mechanisms and designs ${ }^{1-3}$. These mechanisms include self-propulsion of chemical micromotors through catalytic decomposition of a chemical present in the surrounding medium by catalytic metals $\left(\mathrm{Pt}, \mathrm{MnO}_{2}, \mathrm{Pd} \text { and } \mathrm{Ag}\right)^{4,5}$ or enzymes ${ }^{6-9}$, as well as micromotors externally actuated via ultrasound ${ }^{10,11}$, magnetic ${ }^{12-15}$, and electric fields ${ }^{16,17}$. Also, significant progress has recently been made in biohybrid micromotors which use living cells for propulsion and the manipulation of synthetic materials at the micro- and nanoscale ${ }^{18-24}$. Based on their different functionalities ${ }^{1,25,26}$ micro/nanomotors present copious applications in fields, as diverse as nanomedicine ${ }^{27-32}$, sensing ${ }^{33-35}$, and environmental remediation ${ }^{36-38}$. In nanomedicine, synthetic micro/nanomotors have been used for drug transportation and delivery ${ }^{39-41}$, biological sensing ${ }^{42-46}$, cell transport ${ }^{47,48}$, as well as cell ${ }^{40,49,50}$ and tissue penetration $^{51,52}$, highlighting their great potential for developing smart drug delivery systems, as well as their efficacy in sensing and microsurgical applications ${ }^{31,53-55}$.

Many challenges remain before micromotors can safely be deployed in vivo, and only few applications using live animals ${ }^{31,32}$ or in vitro models have been reported ${ }^{54-59}$. One of the major obstacles that needs to be overcome for in vivo use, is the difficulty of tracking micromotors inside the living body ${ }^{60}$, which is not only desirable to be able to gauge their distribution, elimination, and organ or tissue targeting, but it is a necessary requirement for the development of precise and reliable micromotor control and navigation systems.

The use of current optical microscope-based $2 \mathrm{D}$ and $3 \mathrm{D}^{61,62}$ techniques for whole-body in vivo imaging is limited to superficial areas, just below the skin or the surface of internal organs and cavities, because visible light has an inherently low capacity for penetrating biological tissue. Ultrasound imaging can 
penetrate deeper into the living tissue and offers another possibility for the real-time tracking of micromotors. Due to its reliance on the presence of, not only gradients in acoustic impedance, but also structures larger than the sonographic detection limit, this method can only be used to track micro/nanomotors that move via the bubble-propulsion mechanism, as only the trail of expelled microbubbles is detectable with this approach ${ }^{63,64}$. Other high-resolution in vivo imaging techniques employed for micromotor tracking, such as Computed Tomography (CT) or Magnetic Resonance Imaging (MRI) ${ }^{54}$, show only sub-optimal sensitivities, that render the extraction of reliable information, with regard to the actual concentration of the species of interest, extremely challenging ${ }^{65}$. Alternatively, the incorporation of positron or gamma emitters into micromotors may constitute an ideal strategy that allows micromotors to be tracked in vivo using nuclear imaging techniques, e.g., through positron emission tomography (PET) or single photon emission computed tomography (SPECT). These imaging techniques offer poor morphological information and are frequently used in combination with anatomical techniques such as computed tomography (CT). They rely on the detection of gamma rays, which are either directly emitted by gamma-emitting isotopes (SPECT), or generated as a consequence of the positron-electron annihilation process (PET). Nuclear imaging techniques are minimally invasive, ultra-sensitive, and allow for a time-resolved and quantitative determination of the amount of labeled species at the whole-body level, as the amount of emitted radioactivity is directly proportional to the labelled species concentration. Additionally, gamma rays possess a high penetration capacity for biological tissue, enabling PET and SPECT methods to upscale easily from small experimental animals to humans, without encountering any significant issues related to tissue-attenuation. Both PET and SPECT, in combination with CT, are widely utilized in clinical settings, where they serve for the early diagnosis and the evaluation of patient responses to therapy for a variety of diseases. While they have also been widely used for the radiolabeling and tracking of different nano- and micro-particles ${ }^{66,67}$, they have never been applied to the visualization of self-propelled micromotors. 
Here, we demonstrate that micromotors can be tracked in cylindrical phantoms using PET-CT. We used catalytic self-propelled micromotors containing a surface layer of gold as a model of an active system. The gold layer allows for radiolabeling using the positron emitting isotope ${ }^{124}$ I (half-life: 4.2 days). Two identical linear phantoms were prepared to examine the suitability of PET-CT for tracking the location of micromotors (both self-propelled and inert) at a macroscopic level. We observed that PET-CT could accurately track the location of swarming micromotors, providing quantitative information on the temporal evolution of their spatial distribution within the phantoms. The PET-CT results were in good agreement with tracking observations obtained using optical microscopy. In summary, our work demonstrates that PET-CT is capable of visualizing the movements of micromotors on real time, and could eventually be used for the in vivo tracking of swarming micromotors to aid in the development of biomedical applications.

\section{RESULTS AND DISCUSSION}

Self-propelled, gold coated micromotors (Au-micromotors) were synthesized using a common templatedirected electrodeposition protocol ${ }^{4}$, using metal evaporation to coat one side with gold for their radiolabeling. The tubular structure of the micromotors consists of an electrodeposited polymer (Poly(3,4ethylenedioxythiophene or PEDOT) layer and an inner layer made up of platinum (Pt). Au-micromotors were characterized using scanning electron microscopy (SEM) and transmission electron microscopy (TEM), coupled with energy-dispersive X-ray spectroscopy (EDXS) (Figure 1). Our micromotors had a conical shape and an average length of $12 \pm 2 \mu \mathrm{m}(\mathrm{n}=10)$ (Figure 1A-C). The Au surface coating becomes visible in SEM images due to its higher conductivity compared to the underlying polymer (Figure 1B). TEM illustrates the inner conical cavity of a micromotor (Figure 1C) containing Pt, which catalytically decomposes hydrogen peroxide $\left(\mathrm{H}_{2} \mathrm{O}_{2}\right)$ into oxygen and water, thereby producing the oxygen micro-bubbles, that, upon their release from the inner cavity, provide the propulsion mechanism for the micromotors (Figure 1F). The presence of gold on the surface of the micromotor was confirmed using a mapping (Figure 1D) and point analysis (Figure 1E) of the elements present along the transverse axis. 
The signal corresponding to gold could be observed over the entire surface and was especially intense at the edge of the micromotors (point 3 in Figure 1D and 1E), while platinum becomes increasingly abundant as we move away from the edge (Figure 1D and 1E).

The synthesized Au-micromotors were radiolabeled on their gold surface via chemisorption of Iodine-124 (Figure 2A). This approach has been successfully applied for the radiolabeling of gold nanoparticles (AuNPs) $)^{68}$ and gold nanorods (AuNRs) ${ }^{69}$, albeit using different radioisotopes of iodine. Due to the highaffinity binding between gold and iodine, radiochemical yields of $76 \% \pm 4 \%$ (non-decay corrected) could be achieved in short reaction times $(t=10 \mathrm{~min})$. Specific activity at the end of the synthesis was estimated to be $222 \mathrm{MBq} / \mathrm{mg}$. Stability studies showed that $>98 \%$ of the radioactivity was still attached to the micromotors after immersing them in sodium dodecyl sulfate (SDS) solution for 1 hour. Equivalent results were obtained in rat and mouse plasma, and also in $\operatorname{SDS}(1.0 \% \mathrm{w} / \mathrm{v}) / \mathrm{H}_{2} \mathrm{O}_{2}(1.5 \% \mathrm{v} / \mathrm{v})$ solution. In all cases, the fraction of radioactivity attached to the micromotors was $>90 \%$ after 24 hours, pointing to the suitability of the labeling strategy to approach future in vivo studies.

We prepared two identical L-shaped glass phantoms with an internal diameter of $4 \mathrm{~mm}$ as recipients for the micromotors (Figure S1). Their length was kept at $5 \mathrm{~cm}$ (1), thus just below the axial field of view of the PET-CT scanner, so as to enable dynamic 1-bed position imaging (Figure 2B). One of the phantoms was filled with SDS $(1.0 \% \mathrm{w} / \mathrm{v})$ solution (phantom 1) and the second with SDS $(1.0 \% \mathrm{w} / \mathrm{v}) / \mathrm{H}_{2} \mathrm{O}_{2}(1.5 \%$ v/v) solution (phantom 2) (Figure 2B). The micromotors introduced into the solution containing $\mathrm{H}_{2} \mathrm{O}_{2}$ (Figure 2B, 2) started self-propelling immediately after addition; hence, special efforts were made to minimize the elapsed time between sample seeding and image acquisition. Immediately after seeding the micromotors, dynamic PET images were acquired, followed by a CT scan (cf. Figure 2C) for proper localization of the radioactive signal after image reconstruction and co-registration. 
Figure 3 shows PET images acquired at different times after seeding the samples, all of them coregistered with their corresponding CT image (see Figure S2 and Video S1 for complete series). Taking into account that the temporal resolution of PET-CT is rather limited, a compromise had to be made between acquisition time per frame and sensitivity. In our case, we acquired two short frames (frames 1 and 2; duration: 1 minute each), followed by four $2 \mathrm{~min}$ frames and one final $5 \mathrm{~min}$ frame (see Table S1). The total duration of the imaging study was 15 minutes.

Within the first minute after seeding (Frame 1 in Figure 3A), most of the radioactivity remained concentrated at the initial seeding location for both phantoms, i.e., irrespective of the presence of hydrogen peroxide. After about 4-6 minutes (Frame 4 in Figure 3A), the movement of the micromotors in $\mathrm{SDS} / \mathrm{H}_{2} \mathrm{O}_{2}$ (phantom 2 in Figure 3A) becomes visible as the radioactive signal starts to spread out along the length of the tube, while in phantom 1, containing the inert solution, the signal still remained stationary, maintaining the same spatial distribution as in Frame 1. Interestingly, after about 10 to 15 minutes (Frame 7 in Figure 3A), the radioactive signal in phantom 1 had only become slightly blurred compared to the start of the measurements, while a large amount of the mobile micromotors in phantom 2 had travelled to the far end of their tube. This movement also becomes visible if we plot the amount of radioactivity (normalized to the maximum) along the length of the phantom tubes over time (Figure 3B). Clearly, the singular peak present in Frame 1 for both phantoms starts to spread for phantom 2, containing the mobile micromotors (see also the animated GIF in the electronic supplementary material), while it remains relatively stationary for the inert micromotors in phantom 1. It is worth noting, that the initial peak in phantom 2 is wider than for their inert counterparts in phantom 1, indicating that the mobile micromotors had already begun to move during the acquisition of the first frame (duration $=1$ minute).

We divided phantom 2 into three regions of interest (color coded as red, blue, and green in Figure 3B) and determined the amount of radioactivity present in each region, thus yielding histograms for the mobile micromotors for each point in time (Figure 3C). In Frame 1, most of the activity (94.4\%) was contained in the first region containing the seeding location $(0.0-1.5 \mathrm{~cm})$. In Frame 4, 33.7\% of the micromotors had 
reached the mid region, while in Frame 7, more than 50\% of the micromotors had left the initial seeding region and were found either in the mid $(12.2 \%)$ or end sections $(39.8 \%)$ of the tube.

For validation purposes, we repeated the experiment from Figure 3 acquiring optical images of the bubbles and micromotor trajectories (Figure 4). Similar phantoms to those used in the PET-CT experiments were prepared (Figure 4 and S1) and placed on a flat surface. As a control, we immersed an immobilized Pt wire into one of the phantoms and tracked the temporal evolution of the bubble pattern emitted by the Pt wire (Figure 4A), comparing them to the bubble distributions produced by mobile micromotors (Figure 4B). As expected, even after $8 \mathrm{~min}$, most of the $\mathrm{O}_{2}$ bubbles produced by the immobilized platinum wire had remained near the wire's location (Figure 4A), while the $\mathrm{O}_{2}$ bubbles released by the mobile micromotors were increasingly spread out along the length of the phantom tube as time progressed (Figure 4B), which indicates a migration of some micromotors towards the far end of the phantom. This direct visual observation seems to corroborate the results obtained from PET-CT imaging (Figure 3). Due to their initial random orientation, micromotors either migrate towards the far end of the phantom tube, or they remain trapped at their original location. Video $\mathbf{S 2}$ follows one example trajectory where the self-propelled micromotor follows a helical trajectory. Although we observed the majority of micromotors following a helical trajectory, for some micromotors the walls of the phantom acted like a guardrail, forcing them onto a more linear trajectory along the phantom wall. These two kinds of trajectories had previously been observed for spherical and tubular micromotors ${ }^{70,71}$. We estimated the speed of Au-micromotors, based on the measured trajectories, and obtained values of $194.2 \pm 13.5 \mu \mathrm{m} / \mathrm{s}$ (16.2 body lengths/s). For straight line travel, this would yield a minimum time of about 4.5 minutes to traverse the $5 \mathrm{~cm}$ long tube. However, due to their random initial orientation, helical trajectories, and occasional contact with the phantom wall, the average travel time for micromotors would be considerably higher. Our results showed that after 10 to 15 minutes, about $40 \%$ of micromotors had reached the region at the far end of the tube, yielding an effective longitudinal velocity component of about $56-83 \mu \mathrm{m} / \mathrm{s}$. 
These results, obtained using an optical microscope, were qualitatively in good agreement with results obtained from the imaging of swarms using PET-CT.

\section{CONCLUSIONS}

Imaging of micromotors with techniques other than optical approaches remains relatively unexplored. We provided a proof of concept, demonstrating that positron-emission tomography (PET) in combination with X-Ray Computed Tomography (CT) can be used to quantitatively track swarms of bubble-propelled tubular micromotors. We obtained a good qualitative agreement between the locations inferred from PET imaging and the location information obtained from direct visual tracking of the emitted micro-bubbles using an optical microscope. Although the initial orientation of micromotors in this study was random, methods for controlling directionality such as magnetic fields, local gradients, or physical pathways could be used in the near future to orient and thus more accurately guide micromotors to precise locations.

In this context, PET-CT imaging could be a promising technique for imaging large amounts of micromotors moving in confined channels. We believe, this study represents a step forward towards the tracking of micromotors using current medical imaging technology for the in vivo applications of micromotors in the not so distant future.

\section{MATERIALS AND METHODS}

\section{Micromotors synthesis and characterization}

\section{Materials and reagents}

3,4-Ethylenedioxythiophene (EDOT) and sodium dodecyl sulfate (SDS) were purchased from SigmaAldrich (Germany). Hydrogen peroxide 30\%, potassium nitrate, methylene chloride and ethanol were purchased from Merck (Germany). Ultrapure water (Millipore Corporation, USA) was used for the preparation of all aqueous solutions. 


\section{Synthesis of Au-PEDOT/Pt based micromotors}

The PEDOT/Pt microtubes were prepared using a common template-directed electrodeposition protocol. ${ }^{4}$ A cyclopore polycarbonate membrane, containing $2 \mathrm{~mm}$ maximum diameter conical-shaped micropores (catalog no. 7060-2511; Whatman, Maidstone, UK), was employed as a template. A $80 \mathrm{~nm}$ gold film was first sputtered (sputter system MED020 Bal-Tec) on one side of the porous membrane to serve as a working electrode. Sputtering was performed at room temperature under argon environment at $5 \times 10^{-2}$ Torr and $60 \mathrm{~mA}$ current. A Pt wire and $\mathrm{Ag} / \mathrm{AgCl}$ with $3 \mathrm{M} \mathrm{KCl}$ were used as counter and reference electrodes, respectively during the electrodeposition in gold sputtered membrane. The membrane was then assembled in a plating cell with aluminum foil serving as the contact for gold side of the membrane and the working electrode. PEDOT microtubes were electropolymerized for a total charge of $0.06 \mathrm{C}$ at +0.80 V from a plating solution which contains $15 \mathrm{mM}$ EDOT monomer, $100 \mathrm{mM}$ SDS and $7.5 \mathrm{mM}$ $\mathrm{KNO}_{3}$. Then, the metallic layer of Pt was deposited from Pt plating solution (Platinum TP; Technic Deutschland $\mathrm{GmbH})$. The catalytic inner Pt layer was deposited galvanostatically at $-2 \mathrm{~mA}$ for $600 \mathrm{~s}$. To release the microengines from the template, the sputtered gold layer was completely removed by polishing with $5 \mu \mathrm{m}$ alumina slurry. The membrane was then dissolved in methylene chloride for 10 min to completely release the microtubes. Finally, microengines were washed repeatedly with methylene chloride, followed by washing three times with ethanol.

$20 \mu \mathrm{L}$ of the previously synthesized micromotors in ethanol were spread on a glass slide and dried for 2 hours at room temperature. Then, a $10 \mathrm{~nm}$ gold film was sputtered (sputter system MED020 Bal-Tec) on the spreaded micromotors using $\mathrm{XYZ}$ sputter at room temperature under argon environment of $5 \times 10^{-2}$ Torr and $60 \mathrm{~mA}$ current. To obtain the one side sputtered micromotors they were sonicated in ethanol during $5 \mathrm{~min}$ to release them from the glass slide. Afterward, Au-PEDOT/Pt micromotors were washed twice with ethanol and ultrapure water $(18.2 \mathrm{MU} \mathrm{cm})$ and collected by centrifugation at $7000 \mathrm{rpm}$ for 3 min after each wash. They were storage in ultrapure water at room temperature. 


\section{Equipment}

Template electrochemical deposition of microtubes was carried out with using a potentiostat (AUT50101, Metrohm Autolab B.V.). The software used for the electrochemical depositions was NOVA 1.10. An inverted optical microscope (Leica DMI3000B), coupled with a 10X, 20X, 40X and 63X objectives, along with a Leica digital camera DFC3000G with LAS V4.5 soft-ware, was used for capturing movies. Scanning electron microscope (SEM, E-beam-SEM Ultra 55 Zeiss) and transmission electron microscopy (TEM, JEOL JEM-2100F- HAADF, $200 \mathrm{kV}$ ) coupled with Energy dispersive X-ray detector (EDXS, Oxford INCA system) analysis were used for the micromotors characterization. Origin Pro 2016, Microsoft Excel 2016 and INCA software were used for the analysis of the experimental data.

\section{PET-CT for imaging of Micromotors}

\section{Materials}

$\left.{ }^{[24} \mathrm{I}\right] \mathrm{NaI}$ (solution in $0.02 \mathrm{M} \mathrm{NaOH}$ ) was purchased from Perkin Elmer. Sodium dodecyl sulfate ( $\left.\geq 98.0\right)$ and hydrogen peroxide solution (30\% in water) were purchased from Sigma-Aldrich. Ultrapure water $(18 \mathrm{M} \Omega \mathrm{cm})$ was generated using a Milli-Q system (Millipore, Bedford, MA, USA).

\section{Radiolabelling of gold nanomotors with Iodine-124}

The radioiodination of the gold nanomotors was performed by incubation with $\left[{ }^{124} \mathrm{I}\right] \mathrm{NaI}$. In a typical experiment, $50 \mu \mathrm{L}$ of gold nanomotors solution $\left(1 \mathrm{mg} / \mathrm{mL}\right.$ ) and $30 \mu \mathrm{L}$ of $\left[{ }^{124} \mathrm{I}\right] \mathrm{NaI}$ (approximately 15 $\mathrm{MBq}$ ) were incubated at $25^{\circ} \mathrm{C}$ for 10 minutes. After incubation, the crude material was purified by centrifugation (10 min, $13400 \mathrm{rpm}$ ). The resulting precipitate was washed three times with $1 \%$ SDS solution to remove unreacted ${ }^{124} \mathrm{I}$ species, and the amount of radioactivity in the pellet, the supernatant and the washings were determined in a dose calibrator (CPCRC-25R, Capintec Inc., NJ, USA). Finally, the gold micromotors were resuspended in $1 \%$ SDS solution $(100 \mu \mathrm{L})$. Radiochemical yield (expressed in 
percentage) was calculated as the ratio between the amount of radioactivity in the resuspended fraction and the starting amount of radioactivity.

\section{Stability studies}

The radiochemical stability of the radiolabelled nanomotors was assessed by incubation in $1 \%$ SDS solution, $\operatorname{SDS}(1.0 \% \mathrm{w} / \mathrm{v}) / \mathrm{H}_{2} \mathrm{O}_{2}(1.5 \% \mathrm{v} / \mathrm{v})$ solution, and rat and mouse plasma. At different time points $(10,20,30,40$ and 60 min and 24 hours), samples were withdrawn, and the amount of radioactivity was measured. After incubation, the nanomotors were separated by centrifugation (10 min, $13400 \mathrm{rpm})$. The resulting precipitate was washed twice with $1 \%$ SDS solution, and the amount of radioactivity in the pellet, the supernatant and the washings were determined in a dose calibrator (CPCRC-25R, Capintec Inc., NJ, USA). The radiochemical stability was calculated as the percentage of radioactivity in the pellet with respect to the total amount of radioactivity (pellet + filtrate + washings).

\section{Imaging studies}

Imaging studies were conducted using positron emission tomography (PET) in combination with computerized tomography (CT) using an eXplore Vista-CT small animal PET-CT system (GE Healthcare). Two identical L-shaped glass tubes were prepared (see Figure S1a for scheme). The tubes were assembled as depicted in Figure S1b (top view) and filled with 1\% aqueous sodium dodecyl sulfate (SDS) solution and a 1:1 mixture of $1 \%$ SDS and 1.5\% aqueous solution of hydrogen peroxide, respectively.

A sample of labelled nanomotors $(10 \mu \mathrm{L}$, approximately $1 \mathrm{MBq}$ ) was introduced in each of the tubes (see red and green crosses in Figure S1b for seeding location). The tubes were rapidly positioned in the center of the field of view of the scanner, and dynamic scans were acquired in the 400-700 KeV energetic window (one bed position; frames: $2 \times 1 \mathrm{~min}, 4 \times 2 \mathrm{~min}, 1 \times 5 \mathrm{~min}$; total duration: $15 \mathrm{~min}$; see table $\mathrm{S} 1$ for detailed information about timing for each frame). After finalizing the PET acquisition, a CT scan was also carried out to unambiguously position the radioactive signal and to generate the attenuation map to be used during image reconstruction. 
PET images were reconstructed using the 2D-OSEM algorithm ( 2 iterations, 16 subsets) into $175 \times 175 \times 61$ arrays with a voxel size of $0.3875 \times 0.3875 \times 0.775 \mathrm{~mm}$ and were corrected for decay, scatter and random events. The CT images were reconstructed using a cone-beam Feldkamp algorithm into 262x262x688 arrays with a voxel size of $0.246 \mathrm{~mm}^{3}$. Images were analyzed using PMOD image analysis software. With that aim, coronal projections of the PET images were generated for each temporal frame and axial activity profiles along the geometric center of the tubes (see Figure S1b, dotted line) were generated. The values were finally normalized to maximum values for each tube and frame.

\section{ACKNOWLEDGEMENTS}

The research leading to these results has received funding from the European Research Council under the European Union's Seventh Framework Program (FP7/20072013)/ERC grant agreement no. 311529 (LTNRBS) and the Alexander von Humboldt Foundation (D. V.). D. V. acknowledges financial support from the European Commission under Horizon 2020's Marie Skłodowska-Curie Actions COFUND scheme [Grant Agreement no. 712754] and by the Severo Ochoa program of the Spanish Ministry of Economy and Competitiveness [Grant SEV-2014-0425 (2015-2019)]. S.S. wishes to thank the Spanish MINECO for grants CTQ2015-68879-R (MICRODIA) and CTQ2015-72471-EXP (Enzwim). J.L. wishes to thank the Spanish MINECO (project MAT2013-48169-R) and the Department of education, language politics, and culture from Basque regional government (project PI-2014-1-90). IBEC group thanks the CERCA Programme / Generalitat de Catalunya.

\section{AUTHOR INFORMATION}

\section{Corresponding Authors:}

*Email: ssanchez@ibecbarcelona.eu

\section{ORCID}

D. Vilela: 0000-0001-5005-7070

U. Cossio: 0000-0002-3248-8683

J. Parmar: 0000-0001-7561-7417 
A.M. Martínez-Villacorta: 0000-0002-3199-0479

V. Gómez-Vallejo: 0000-0003-3995-9596

J. Llop: 0000-0002-0821-9838

S. Sanchez 0000-0002-5845-8941

\section{REFERENCES}

(1) Guix, M.; Mayorga-Martinez, C. C.; Merkoçi, A. Nano/Micromotors in (Bio)chemical Science Applications. Chem. Rev. 2014, 114, 6285-6322.

(2) Sánchez, S.; Soler, L.; Katuri, J. Chemically Powered Micro- and Nanomotors. Angew. Chemie Int. Ed. 2015, 54, 1414-1444.

(3) Wang, H.; Pumera, M. Fabrication of Micro/Nanoscale Motors. Chem. Rev. 2015, 115, 87048735.

(4) Gao, W.; Sattayasamitsathit, S.; Uygun, A.; Pei, A.; Ponedal, A.; Wang, J.; Claussen, J. C.; Merkoçi, A.; Wang, J.; Lee, S. B. Polymer-Based Tubular Microbots: Role of Composition and Preparation. Nanoscale 2012, 4, 2447.

(5) Safdar, M.; Wani, O. M.; Jänis, J. Manganese Oxide-Based Chemically Powered Micromotors. ACS Appl. Mater. Interfaces 2015, 7, 25580-25585.

(6) Ma, X.; Hortelão, A. C.; Patiño, T.; Sánchez, S. Enzyme Catalysis To Power Micro/Nanomachines. ACS Nano 2016, 10, 9111-9122.

(7) Ma, X.; Jannasch, A.; Albrecht, U.-R.; Hahn, K.; Miguel-López, A.; Schäffer, E.; Sánchez, S. EnzymePowered Hollow Mesoporous Janus Nanomotors. Nano Lett. 2015, 15, 7043-7050.

(8) Ma, X.; Hortelao, A. C.; Miguel-López, A.; Sánchez, S. Bubble-Free Propulsion of Ultrasmall 
Tubular Nanojets Powered by Biocatalytic Reactions. J. Am. Chem. Soc. 2016, 138, 13782-13785.

(9) Dey, K. K.; Zhao, X.; Tansi, B. M.; Méndez-Ortiz, W. J.; Córdova-Figueroa, U. M.; Golestanian, R.; Sen, A. Micromotors Powered by Enzyme Catalysis. Nano Lett. 2015, 15, 8311-8315.

(10) Garcia-Gradilla, V.; Orozco, J.; Sattayasamitsathit, S.; Soto, F.; Kuralay, F.; Pourazary, A.; Katzenberg, A.; Gao, W.; Shen, Y.; Wang, J. Functionalized Ultrasound-Propelled Magnetically Guided Nanomotors: Toward Practical Biomedical Applications. ACS Nano 2013, 7, 9232-9240.

(11) Ahmed, D.; Baasch, T.; Jang, B.; Pane, S.; Dual, J.; Nelson, B. J. Artificial Swimmers Propelled by Acoustically Activated Flagella. Nano Lett. 2016, 16, 4968-4974.

Jang, B.; Gutman, E.; Stucki, N.; Seitz, B. F.; Wendel-García, P. D.; Newton, T.; Pokki, J.; Ergeneman, O.; Pané, S.; Or, Y.; Nelson, B. J. Undulatory Locomotion of Magnetic Multilink Nanoswimmers. Nano Lett. 2015, 15, 4829-4833.

(13) Chen, X.-Z.; Hoop, M.; Shamsudhin, N.; Huang, T.; Özkale, B.; Li, Q.; Siringil, E.; Mushtaq, F.; Di Tizio, L.; Nelson, B. J.; Salvador P. Hybrid Magnetoelectric Nanowires for Nanorobotic Applications: Fabrication, Magnetoelectric Coupling, and Magnetically Assisted In Vitro Targeted Drug Delivery. Adv. Mater. 2017, 29, 1605458.

(14) Mark, A. G.; Gibbs, J. G.; Lee, T.-C.; Fischer, P. Hybrid Nanocolloids with Programmed ThreeDimensional Shape and Material Composition. Nat Mater 2013, 12, 802-807.

(15) Maier, A. M.; Weig, C.; Oswald, P.; Frey, E.; Fischer, P.; Liedl, T. Magnetic Propulsion of Microswimmers with DNA-Based Flagellar Bundles. Nano Lett. 2016, 16, 906-910.

(16) Calvo-Marzal, P.; Sattayasamitsathit, S.; Balasubramanian, S.; Windmiller, J. R.; Dao, C.; Wang, J. Propulsion of Nanowire Diodes. Chem. Commun. 2010, 46, 1623-1624. 
(17) Loget, G.; Kuhn, A. Electric Field-Induced Chemical Locomotion of Conducting Objects. Nat. Commun. 2011, 2, 535.

(18) Stanton, M. M.; Simmchen, J.; Ma, X.; Miguel-López, A.; Sánchez, S. Biohybrid Janus Motors Driven by Escherichia Coli. Adv. Mater. Interfaces 2016, 3, 1500505.

(19) Stanton, M. M.; Park, B.-W.; Miguel-López, A.; Ma, X.; Sitti, M.; Sánchez, S. Biohybrid Microtube Swimmers Driven by Single Captured Bacteria. Small 2017, 13, 1603679.

(20) Medina-Sánchez, M.; Schwarz, L.; Meyer, A. K.; Hebenstreit, F.; Schmidt, O. G. Cellular Cargo Delivery: Toward Assisted Fertilization by Sperm-Carrying Micromotors. Nano Lett. 2016, 16, $555-561$.

(21) Zhuang, J.; Park, B.-W.; Sitti, M. Propulsion and Chemotaxis in Bacteria-Driven Microswimmers. Adv. Sci. 2017, 4, 1700109.

(22) Felfoul, O.; Mohammadi, M.; Taherkhani, S.; de Lanauze, D.; Zhong Xu, Y.; Loghin, D.; Essa, S.; Jancik, S.; Houle, D.; Lafleur, M.; Gaboury,L.; Tabrizian, M.; Kaou, N.; Atkin,M.; Vuong, T.; Batist, G.; Beauchemin,N.; Martel, S. Magneto-Aerotactic Bacteria Deliver Drug-Containing Nanoliposomes to Tumour Hypoxic Regions. Nat. Nanotechnol. 2016, 11, 941-947.

(23) Park, B.-W.; Zhuang, J.; Yasa, O.; Sitti, M. Multifunctional Bacteria-Driven Microswimmers for Targeted Active Drug Delivery. ACS Nano 2017, 11, 8910-8923.

(24) Stanton, M. M.; Park, B.-W.; Vilela, D.; Bente, K.; Faivre, D.; Sitti, M.; Sanchez, S. Magnetotactic Bacteria Powered Biohybrids Target E. Coli Biofilms. ACS Nano 2017, 11, 9968-9978.

(25) Katuri, J.; Ma, X.; Stanton, M. M.; Sánchez, S. Designing Micro- and Nanoswimmers for Specific Applications. Acc. Chem. Res. 2017, 50, 2-11. 
(26) Li, J.; Esteban-Fernández de Ávila, B.; Gao, W.; Zhang, L.; Wang, J. Micro/nanorobots for Biomedicine: Delivery, Surgery, Sensing, and Detoxification. Sci. Robot. 2017, 2, eaam6431.

(27) Ma, X.; Sánchez, S. Self-Propelling Micro-Nanorobots: Challenges and Future Perspectives in Nanomedicine. Nanomedicine 2017, 12, 1363-1367.

(28) Stanton, M. M.; Sánchez, S. Pushing Bacterial Biohybrids to In Vivo Applications. Trends Biotechnol. 2017, 35, 910-913.

(29) Abdelmohsen, L. K. E. A.; Peng, F.; Tu, Y.; Wilson, D. A. Micro- and Nano-Motors for Biomedical Applications. J. Mater. Chem. B 2014, 2, 2395-2408.

(30) Patra, D.; Sengupta, S.; Duan, W.; Zhang, H.; Pavlick, R.; Sen, A. Intelligent, Self-Powered, Drug Delivery Systems. Nanoscale 2013, 5, 1273-1283.

(31) de Ávila, B. E.-F.; Angsantikul, P.; Li, J.; Angel Lopez-Ramirez, M.; Ramírez-Herrera, D. E.; Thamphiwatana, S.; Chen, C.; Delezuk, J.; Samakapiruk, R.; Ramez, V.; Obonyo, M.; Zhang, L.; Wang, J. Micromotor-Enabled Active Drug Delivery for in Vivo Treatment of Stomach Infection. Nat. Commun. 2017, 8, 272.

(32) Gao, W.; Dong, R.; Thamphiwatana, S.; Li, J.; Gao, W.; Zhang, L.; Wang, J. Artificial Micromotors in the Mouse's Stomach: A Step toward in Vivo Use of Synthetic Motors. ACS Nano 2015, 9, 117123.

(33) Jurado-Sánchez, B.; Escarpa, A. Milli, Micro and Nanomotors: Novel Analytical Tools for RealWorld Applications. TrAC Trends Anal. Chem. 2016, 84, 48-59.

(34) Jurado-Sánchez, B.; Escarpa, A. Janus Micromotors for Electrochemical Sensing and Biosensing Applications: A Review. Electroanalysis 2017, 29, 14-23. 
(35) Chałupniak, A.; Morales-Narváez, E.; Merkoçi, A. Micro and Nanomotors in Diagnostics. Adv. Drug Deliv. Rev. 2015, 95, 104-116.

(36) Soler, L.; Sánchez, S. Catalytic Nanomotors for Environmental Monitoring and Water Remediation. Nanoscale 2014, 6, 7175-7182.

(37) Gao, W.; Wang, J. The Environmental Impact of Micro/Nanomachines: A Review. ACS Nano 2014, 8, 3170-3180.

(38) Moo, J. G. S.; Pumera, M. Chemical Energy Powered Nano/Micro/Macromotors and the Environment. Chem. - A Eur. J. 2015, 21, 58-72.

(39) Ma, X.; Hahn, K.; Sanchez, S. Catalytic Mesoporous Janus Nanomotors for Active Cargo Delivery. J. Am. Chem. Soc. 2015, 137, 4976-4979.

(40) Esteban-Fernández de Ávila, B.; Angell, C.; Soto, F.; Lopez-Ramirez, M. A.; Báez, D. F.; Xie, S.; Wang, J.; Chen, Y. Acoustically Propelled Nanomotors for Intracellular siRNA Delivery. ACS Nano 2016, 10, 4997-5005.

(41) Sattayasamitsathit, S.; Kou, H.; Gao, W.; Thavarajah, W.; Kaufmann, K.; Zhang, L.; Wang, J. Fully Loaded Micromotors for Combinatorial Delivery and Autonomous Release of Cargoes. Small 2014, 10, 2830-2833.

(42) Esteban-Fernández de Ávila, B.; Martín, A.; Soto, F.; Lopez-Ramirez, M. A.; Campuzano, S.; Vásquez-Machado, G. M.; Gao, W.; Zhang, L.; Wang, J. Single Cell Real-Time miRNAs Sensing Based on Nanomotors. ACS Nano 2015, 9, 6756-6764.

(43) Vilela, D.; Orozco, J.; Cheng, G.; Sattayasamitsathit, S.; Galarnyk, M.; Kan, C.; Wang, J.; Escarpa, A. Multiplexed Immunoassay Based on Micromotors and Microscale Tags. Lab Chip 2014, 14, 3505. 
(44) de Ávila, B. E.-F.; Zhao, M.; Campuzano, S.; Ricci, F.; Pingarrón, J. M.; Mascini, M.; Wang, J. Rapid Micromotor-Based Naked-Eye Immunoassay. Talanta 2017, 167, 651-657.

(45) Rojas, D.; Jurado-Sánchez, B.; Escarpa, A. "Shoot and Sense" Janus Micromotors-Based Strategy for the Simultaneous Degradation and Detection of Persistent Organic Pollutants in Food and Biological Samples. Anal. Chem. 2016, 88, 4153-4160.

(46) Fu, S.; Zhang, X.; Xie, Y.; Wu, J.; Ju, H. An Efficient Enzyme-Powered Micromotor Device Fabricated by Cyclic Alternate Hybridization Assembly for DNA Detection. Nanoscale 2017, 9, 9026-9033.

(47) Sanchez, S.; Solovev, A. A.; Schulze, S.; Schmidt, O. G. Controlled Manipulation of Multiple Cells Using Catalytic Microbots. Chem. Commun. 2011, 47, 698-700.

(48) Yoshizumi, Y.; Okubo, K.; Yokokawa, M.; Suzuki, H. Programmed Transport and Release of Cells by Self-Propelled Micromotors. Langmuir 2016, 32, 9381-9388.

Campuzano, S.; Orozco, J.; Kagan, D.; Guix, M.; Gao, W.; Sattayasamitsathit, S.; Claussen, J. C.; Merkoçi, A.; Wang, J. Bacterial Isolation by Lectin-Modified Microengines. Nano Lett. 2012, 12, 396-401.

(50) Esteban-Fernández de Ávila, B.; Ramírez-Herrera, D. E.; Campuzano, S.; Angsantikul, P.; Zhang, L.; Wang, J. Nanomotor-Enabled pH-Responsive Intracellular Delivery of Caspase-3: Toward Rapid Cell Apoptosis. ACS Nano 2017, 11, 5367-5374.

(51) Xi, W.; Solovev, A. A.; Ananth, A. N.; Gracias, D. H.; Sanchez, S.; Schmidt, O. G. Rolled-up Magnetic Microdrillers: Towards Remotely Controlled Minimally Invasive Surgery. Nanoscale 2013, 5, 1294-1297. 
(52) Pokki, J.; Parmar, J.; Ergeneman, O.; Torun, H.; Guerrero, M.; Pellicer, E.; Sort, J.; Pané, S.; Nelson, B. J. Mobility-Enhancing Coatings for Vitreoretinal Surgical Devices: Hydrophilic and Enzymatic Coatings Investigated by Microrheology. ACS Appl. Mater. Interfaces 2015, 7, 22018-22028.

(53)

Li, J.; Angsantikul, P.; Liu, W.; Esteban-Fernández de Ávila, B.; Thamphiwatana, S.; Xu, M.; Sandraz, E.; Wang, X.; Delezuk, J.; Gao, W.; Zhang, L.; Wang. J. Micromotors Spontaneously Neutralize Gastric Acid for pH-Responsive Payload Release. Angew. Chemie Int. Ed. 2017, 56, $2156-2161$.

(54) Vonthron, M.; Lalande, V.; Bringout, G.; Tremblay, C.; Martel, S. A MRI-Based Integrated Platform for the Navigation of Micro-Devices and Microrobots. In 2011 IEEE/RSJ International Conference on Intelligent Robots and Systems; IEEE, 2011; pp. 1285-1290.

(55) Ullrich, F.; Bergeles, C.; Pokki, J.; Ergeneman, O.; Erni, S.; Chatzipirpiridis, G.; Pané, S.; Framme, C.; Nelson, B. J. Mobility Experiments With Microrobots for Minimally Invasive Intraocular Surgery. Investig. Opthalmology Vis. Sci. 2013, 54, 2853.

(56) Wu, Z.; Esteban-Fernández de Ávila, B.; Martín, A.; Christianson, C.; Gao, W.; Thamphiwatana, S. K.; Escarpa, A.; He, Q.; Zhang, L.; Wang, J. RBC Micromotors Carrying Multiple Cargos towards Potential Theranostic Applications. Nanoscale 2015, 7, 13680-13686.

(57)

Li, J.; Angsantikul, P.; Liu, W.; Esteban-Fernández de Ávila, B.; Thamphiwatana, S.; Xu, M.; Sandraz, E.; Wang, X.; Delezuk, J.; Gao, W.; Zhang, L.; Wang, J. Micromotors Spontaneously Neutralize Gastric Acid for pH-Responsive Payload Release. Angew. Chemie Int. Ed. 2017, 56, $2156-2161$.

(58) He, W.; Frueh, J.; Hu, N.; Liu, L.; Gai, M.; He, Q. Guidable Thermophoretic Janus Micromotors Containing Gold Nanocolorifiers for Infrared Laser Assisted Tissue Welding. Adv. Sci. 2016, 3, 
1600206.

(59) Srivastava, S. K.; Medina-Sánchez, M.; Koch, B.; Schmidt, O. G. Medibots: Dual-Action Biogenic Microdaggers for Single-Cell Surgery and Drug Release. Adv. Mater. 2016, 28, 832-837.

(60) Medina-Sánchez, M.; Schmidt, O. G. Medical Microbots Need Better Imaging and Control. Nat. News 2017, 545, 406. Nature. 2017 May 24;545(7655):406-408. doi: 10.1038/545406a

(61) Hong, A.; Zeydan, B.; Charreyron, S.; Ergeneman, O.; Pane, S.; Toy, M. F.; Petruska, A. J.; Nelson, B. J. Real-Time Holographic Tracking and Control of Microrobots. IEEE Robot. Autom. Lett. 2017, $2,143-148$.

(62) Glückstad, J. Light-Driven Micro-Robotics with Holographic 3D Tracking. In Proceedings of SPIE 2016, 9845 .

(63) Sanchez, A.; Magdanz, V.; Schmidt, O. G.; Misra, S. Magnetic Control of Self-Propelled Microjets under Ultrasound Image Guidance. In 5th IEEE RAS/EMBS International Conference on Biomedical Robotics and Biomechatronics, $2014,169-174$.

(64) Olson, E. S.; Orozco, J.; Wu, Z.; Malone, C. D.; Yi, B.; Gao, W.; Eghtedari, M.; Wang, J.; Mattrey, R. F. Toward in Vivo Detection of Hydrogen Peroxide with Ultrasound Molecular Imaging. Biomaterials 2013, 34, 8918-8924.

(65) Cunha, L.; Horvath, I.; Ferreira, S.; Lemos, J.; Costa, P.; Vieira, D.; Veres, D. S.; Szigeti, K.; Summavielle, T.; Máthé, D.; Metello, L. F. Preclinical Imaging: An Essential Ally in Modern Biosciences. Mol. Diagn. Ther. 2014, 18, 153-173.

(66) Llop, J.; Gómez-Vallejo, V.; Gibson, N. Quantitative Determination of the Biodistribution of Nanoparticles: Could Radiolabeling Be the Answer? Nanomedicine 2013, 8, 1035-1038. 
(67) Llop, J.; Gomez-Vallejo, V.; Neil, G. Isotopes in Nanoparticles: Fundamentals and Applications. Pan Stanford 2016.

(68) Shao, X.; Agarwal, A.; Rajian, J. R.; Kotov, N. A.; Wang, X. Synthesis and Bioevaluation of ${ }^{125}$ ILabeled Gold Nanorods. Nanotechnology 2011, 22, 135102.

(69) Eskandari, N.; Yavari, K.; Outokesh, M.; Sadjadi, S.; Ahmadi, S. J. lodine-131 Radiolabeling of Poly Ethylene Glycol-Coated Gold Nanorods for in Vivo Imaging. J. Label. Compd. Radiopharm. 2013, $56,12-16$.

(70) Sanchez, S.; Ananth, A. N.; Fomin, V. M.; Viehrig, M.; Schmidt, O. G. Superfast Motion of Catalytic Microjet Engines at Physiological Temperature. J. Am. Chem. Soc. 2011, 133, 14860-14863.

(71) Simmchen, J.; Katuri, J.; Uspal, W. E.; Popescu, M. N.; Tasinkevych, M.; Sánchez, S. Topographical Pathways Guide Chemical Microswimmers. Nat. Commun. 2016, 7, 10598. 